\title{
Nay or Jain Nyay 1: Existence Inferred from Affirmed
}

\section{Assertions}

\author{
Mahendra Kumar Jain \\ University of Delaware
}

\begin{abstract}
The Jain logic (Nay) addresses concerns elicited by sense experience of observable and measurable reality. Reality is what it is, and it exists independent of the observer. The last Jain Tirthankar Mahaveer suggested that organisms interact with such realities for survival needs and become concerned about the consequences. He suggested a code of conduct for reality-based behaviors to address concerns. Perceptions and impressions provide measures (praman) of information in sense experience, and with other evidence guide choices and decisions to act and bear consequences. Ethical behaviors rooted in reality have desirable consequences, and inconsistent and contradictory behaviors are undesirable consequences. Omniscience (God, Brahm) is discarded as a self-referential ad hoc construct inconsistent and contradictory to real world behaviors. This article is survey of assumptions and models to represent, interpret, and validate knowledge that begins with logical deduction for inference (anuman) based on evidence from sense experience (Jain 2011). Secular and atheistic thrust of thought and practice encourages reasoning and open-ended search with affirmed assertions and independent evidence. Individual identity (atm) emerges with consistent behaviors to overcome fallibility and unreliability by minimizing doubt (Syad-Saptbhangi Nay). The first Tirthankar Rishabh Nath (ca. 2700 BC) suggested that the content (sat) of real and abstract objects and concerns during a change is conserved as the net balance of the inputs and outputs (Tatia 1994). Identity and content of assertions and evidence is also conserved during logical manipulations for reasoning. Each assertion and its negation are to be affirmed with independent evidence, and lack of evidence for presence is not necessarily the evidence for either non-absence or non-existence.
\end{abstract}

Keywords: Saptbhangi Syad Nay, Jain logic, evidence-based inference, quantum logic

\section{Introduction}

Contemporary Jain thought and practice is rooted in earlier ideas from the tradition (Hiraiynna 1932; Roy 1984; Sanghvi 1989). The last and 24th Tirthankar Vardhman Mahaveer (599-527 BC) reorganized earlier ideas and practice, and extended their scope to address social and intellectual concerns of his period. He argued against the (non-human) other-worldly origin of Vedas. He refuted Vedic and Vedantic (post-Vedic and Upnishadic) assertions about the role of the Universal Supreme creator and decision maker that forever is beyond sense experience. Even those who claim to have experienced the divine light describe it in ways that are often inconsistent if not contradictory. It is claimed that apparently random behaviors for all organisms are determined, scripted, and acted upon by what is purported to be omniscient intelligent supreme (potter and

Mahendra Kumar Jain, emeritus professor, Department of Chemistry and Biochemistry, University of Delaware, USA; main research fields: 2D-Interfacial Catalysis and Logic of Doubt in Evidence-Based Inference. Email: mkjain@udel.edu. 
judge). Such objects of faith do not conform to rules of reality. Their behaviors are random at best, and consequences of their presence or absence are indistinguishable.

Mahaveer expressed his ideas in debates with scholars of his time, and further elaborated in discourses with his group. Gautam, a well-respected scholar of Upnishads and expert on rules of debate, is probably the Gautam of Gautam (Nyay) Sutr compiled by Akchpad (ca. 200 BC). At the age of 51, he joined the group of Mahaveer (who was 42) as a discussion leader. Much of what we know about Mahaveer is through answers to queries and concerns of Gautam. These were probably assembled after the death of Mahaveer when Gautam was over 80. This article draws from interpretative translation (with a focus on the content and message rather than linguistic nuances) of works on Nay by Gautam, Samantbhadra (200 AD), Siddhsen Divakar (400 AD), Haribhadra (500 AD), Akalank (700 AD), Manikyanandi (900 AD), and Gunratn (1400 AD). Interpretative translation of these works and my essays on Nay as Jain Nyay are available at http://www.hira-pub.org/nay/index.html.

Gist of the world-view of Mahaveer is that organisms individually and collectively use information from sense experience to identify and address concerns. By trial and error they learn that actions have consequences, which serves as guide for purposeful choices and decisions. For example, choice of fight or flight, or act or not-to-act, as response follows from perceived consequences. Organisms as actor and decision-maker improve quality of life with mutual interdependence and conservation of resources. Humans also use outcome-based strategy for successful behavior (Rogers and Jain 1993). Most people learn such skills and strategies through childhood play where groups also bring out innate sense of fair play. Ability to infer consequences of actions and behaviors comes with added responsibility for live and let live and thrive to maintain integrity of the web of life.

\section{Information from Processed Sense Inputs and Experience}

Sense organs are windows that let in specific information as smell, light, taste, sound or touch about the external world (pratyakch, literally in front of the eyes). Relevance of its meaning and significance to identify and address concerns is interpreted behind the eyes (parokch) by mind (manas). Mind of each individual organism is programmed largely by its experiences during development (sanskar). Perceptions relevant to concerns are scrutinized and interpreted in real time in relation to other experiences and evidence.

Information from fragments of external reality is used for survival needs: food, respiration (air and water), and reproduction. Freedoms to move, express, and communicate are necessary for social order. Evolutionarily developed anatomy of brain for such reasons goes through remarkably similar stages of development in most organisms. The architecture of brain is similar among individuals of a species, and also across the species. Each class goes through similar stages of development during which they learn behaviors characteristic of the class. The brain is hard-wired and programmed to carry out functions of mind under the influences of sense experiences unique to each individual. Sense inputs are interpreted by the architecture of brain for neuromuscular and neuroendocrine regulation of functions for survival needs. Neural networks of brain also serve functions of mind that influence virtually all life processes.

Sense organs sample reality in real time. Macro-level perceptions and impressions from sense experience are to be validated by independent evidence including the experiences from the past. Awareness of a fraction of inputs received by sense organ is converted to linear trains of electrical signals. They are filtered and transmitted to different parts of the brain for further processing to instruct different parts of the body for 
coordinated response. Typical response time for sense stimuli is few seconds during which the message may pass through millions of neurons. Sense receptors that initiate the signals are better suited to detect the space and time dependent changes, including gradients, profiles, and movements. For the lack of a better description, signals are interpreted as images by the mind. Both the signals and images have little resemblance to the object they code. Yet the coded information is stored, accessed, retrieved, recalled, manipulated, and processed as a whole or in parts as needed.

Information is often coded as linear sequence of alphabets. Binary digital computers use sequences of 0 (off) and 1 (on) alphabets; genetic information in DNA is coded in sequences of four bases ACGT; protein sequences are made up of 20 amino acids; music compositions use 7 notes; expressions in English use 26 alphabets. In most languages, information about discrete parts of reality is coded in unique order of alphabets (words) and sequences (sentence) separated by spaces and symbols (such as full stop and commas): Words identify objects and actions; word strings assert relations between one or more parts; description and narrative with multiple assertions communicate concerns. In symbolic languages, alphabet sequences and their order communicate all that has been spoken and is likely to be ever said in the future. It is not known how the mental images of the sense experience are stored and processed behind the eyes. It is likely to be a symbolic language of alphabets of signals processed through networks of neurons interconnected through axons and synapses. Parts of the network may be programmed by input signal for specific tasks. Parallel processing in specific regions of brain may occur through 1, 2, or 3 dimensional arrays of activated and inhibitory neurons. Additional information may be coded with threshold, refractoriness, and frequency of action potential as the building block of the signals.

Independently affirmed assertions about an object or concern make up valid proposition and description. Empirical searches identify and interpret objects and concerns in as many ways as possible. Such linear narratives can be skilfully woven into a multi-dimensional tapestry that evokes images and insights. Medium of representation and analytical methods (paintings, music, narratives, descriptions, and mathematics) emphasize certain features of sense experience, while others remain unattended or implicit. Information is there in what is expressed and also in what is left out. Conventions of grammar may facilitate communication of nuances, however successful reasoning requires that all available information and evidence be interpreted and reconciled with rules of logic to track underlying reality. Reasoning requires tools and devices (instruments and procedures) to resolve inconsistencies, reduce uncertainty in inputs, and discard contradictions and self-reference. Inferences from atheistic and secular assumptions are more likely to be consistent with reality inherent in the inputs from the past and also those likely to be encountered in future. Illusions of faith and belief are often beyond boundaries of information and limitations of time. Such ad hoc assumptions ignore reality and distort reasoning.

Human progress is based on use of symbolic languages to represent real world happenings. Abstractions of valid ideas with verified information provide a rigorous basis to scrutinize descriptions of sense experiences. Ideas and thoughts represented in symbolic languages can be shared with others separated by distances and time for mind to mind communication and guide open-ended search. Stronger inferences ultimately become part of shared knowledge. It is at its best if its growth and viability is assured if it can be manipulated, deliberated, communicated, and interpreted in different contexts to explore meaning and significance of experience. Ready access to information in print or on Internet Web has provided platforms for virtually continuous interpretations and refinements. Sciences and technologies are repertoire of results collectively 
verified with rigor and healthy dose of skeptical scrutiny. Even the non-practitioners and skeptics benefit from such knowledge. Its products have done more to improve human condition than all divine insights put together. Personal knowledge at best may benefit the practitioner, and at worse it is like a blind snake in a dark hole.

\section{Reap What You Sow}

All organisms live with bounded rationality of instinctive behaviors, and seek ways to break out of prisons of personal knowledge. Instinctive behaviors encourage fight-or-flight response for survival in niches or in cocoon of own mind. Environments free of external and internal threats facilitate response with ideas and actions that are freely generated, communicated, scrutinized, and validated by trial and error. Affirmed assertions and valid concepts permit reasoning. Decision choices for rational behaviors are explored from parts of sense experience and from willingness to learn from experience of others during childhood play. Rules and strategies emerge by trial and error through empirical search guided by requirement of successful outcome and relevance for future behaviors in practice.

\section{Representation of Sense Experience}

Shatkhandagam by Pushpdant and Bhutbali (37 AD) is the oldest written work from the Jain tradition. "Satprarupana (criteria based representation of reality)" is the first chapter of Shatkhandagam (Saha 1965). It begins with Namokar to acknowledge five groups of people who pave the way to create, prove, scrutinize, teach, and disseminate knowledge to address individual and collective concerns of all. Their empirical approaches empower the practitioners to generate and reorganize ideas, and reshuffle concepts. Consequences and relevance of emerging concerns may be obvious and immediate but lasting changes in the shared knowledge take time to formulate productive ideas verified and validated through practice. Barren ideas invoked with unquestioning faith and beliefs rationalized with personal knowledge are discarded as they become irrelevant.

Namokar acknowledges the source and processes of prior knowledge. Together with the three more steps outlined in the next 22 lines, it makes up the abstract of Satprarupana (representation) part of Shatkhandagam: to collect (margana) information; to search for generalizations (anuyog) to create knowledge; and to improve quality of perceptions (itthi) with knowledge. The second step (margana) is to identify parts of the physical order perceived by the five senses (indiya) and other organs (atiindiya for gravity, pain, and pleasure), or by extrasensory means (anindiya for deduction and inference). In the third step, the criteria based observations are organized and categorized in relation to the generalizations for space, time, and classes of known objects. As is apparent from the abstract of any modern scientific paper, the first three steps acknowledge prior knowledge to organize, categorize, and process information for interpretation. The fourth step is unique to Shatkhandagam. It emphasizes that validity of sense experience for creation and use of knowledge depends on the quality of perception (gunasthan) of the interpreter.

\section{Reality Check: Can It Be Observed?}

Identity of an object or concern is developed from its sense inputs. Assertions about existence of an object or concern are addressed with what, when, where, and how type of questions. Assertions about well-defined parts and relations of the object provide a basis for its representation to facilitate reasoning. Representations are useful to address: Can the object be "seen" by senses? Can such sense experience be described in ways that 
others understand? Are the consequences of the presence of object different from those of its absence? Answers to such curiosity driven questions may be affirmed by independent evidence. Human curiosity about object of concern draws from mental images of sense experiences. Such creative processes underlie discoveries, innovations, and inventions as arts and crafts, toys of technologies, symbolic languages, abstractions, and theories (for example for atomic structure, biological evolution, or relativity of space and time).

\section{Reality Check: Can Its Experience Be Described?}

Comprehension of an object of concern, including its real and abstract parts and their relations, evolves from articulated sense experience by trial and error. Empirical knowledge from fragmented information develops in fits and starts. Here the key assumption is that external reality may appear complex but it is what it is. Its awareness from sense inputs may be complex but never contradictory. Object of concern exists without the observer, however its perceptions from sense experience are largely constructs of the observer's mind. Sense inputs from it are amenable to resolution by human efforts which go long way towards understanding the world around us. Individually and collectively humans share the same reality but each individual interprets world happenings in relation to own experiences. Suitably articulated opinions converge as reality of object or concern is established. The reality of individual mind is in the sense experiences that permit interpretation of the meaning and significance of inputs. Quality of perceptions may therefore influence quality of reasoning with parts captured from sense inputs and representations. Inconsistencies creep in if sense experience is corrupted or not relevant to the concern.

\section{Reality Check: Does Information Make a Difference for Reasoning?}

Explicitly identified content and context of the object or concern facilitate its representation for reasoning. Perceptions from sense experience guide communication and deliberation for validation. Mental images of sense experience expressed as word constructs facilitate search for reality as a shared enterprise to understand assumptions, implications, meaning, and significance of the experience. Successful representations (descriptions, behaviors, practices) of shared experiences become integral part of knowledge. Verified parts and validated relations become part of growing knowledge. It is like a vine that flourishes where ever it finds open space with light, air, nutrients, and support. Hardly anything flourishes under the shade of a Banyan tree. Akalank (ca. $700 \mathrm{AD}$ ) emphasized that sharing well-formed representations encourages growth and viability of knowledge.

Multidimensional searches are necessary because senses are fallible, perceptions can be swayed, recall can be selective, and faulty memory often plays tricks with mind. Sense organs sample and process inputs from reality that is shared by all. Yet articulated sense experiences are open to doubt. Languages facilitate $a$ posteriori processing of mental images to describe, clarify, communicate, deliberate, and scrutinize objects of concern. Relations of identified parts provide insights for interpretation of meaning and significance. Grammar alone does not distinguish facts from fiction. For example, that a green cat jumped from the branch of a red tree and landed on Moon may be grammatically correct and even logically justifiable, but the proposition as a whole is certainly not consistent with realities on Earth. Most of the human activities and concerns are fictions perpetuated and sustained with fear, faith, belief, and other tricks that make people stupid. Virtually all notions of interpretations of sense experiences recognize corrupting influences of expectations, emotions, and desires. Experts as well as novices see what they know or want to see. Also a person with hammer sees only nails. 
Search for the meaning and significance of sense experiences therefore relies on deliberations and interpretations where measured and verified inputs affirmed by independent evidence. Foundation of all viable technologies and ideas is search grounded in reality. Barren ideas perpetuated by faith or fear reappear in many forms such as omniscience, perpetual motion machine, Maxwell demon, kalp-tree, paras-stone (philosopher's stone), or Mida's gold.

\section{Interaction of Time with Physical Reality: What Is Done Cannot Be Undone}

Mathematical as well as ad hoc constructs may treat time as reversible or irreversible to be affirmed by evidence rooted in physical reality. A code of conduct rooted in physical reality builds on the observed and experienced irreversibility of actions and consequences as the basis for behavior modification: Violence, lying, stealing, illicit relations, and possessions have undesirable, unpredictable, unpleasant, and lasting consequences. Inevitable outcomes and consequences are parts of the same reality. As reward or punishment, consequences of actions cannot be undone or forgiven by grace which makes it necessary to consider outcomes before acting. Timing and nature of the outcome may not be predictable but relationship of actions to consequences is statistical, as for smoking to lung cancer. The action-consequence cycle plays out when all dues are paid. The Karma theory calls for avoiding actions that have undesirable outcome and those commit to undesirable binding consequences (karm bandh). There may be second chance but outcome will not change unless actions are modified for desired outcome. Observed outcome of trial and error or even random actions is useful to learn and carry out mid-course corrections. Misplaced faith and beliefs do not offer such opportunities. Repeating the same action with expectation of different outcome is insanity.

\section{Valid Representation}

Anything goes if it works for empirical search by trial and error. Ad hoc beliefs prejudge and moral assumptions prejudice reasoning. Secular and atheistic mindset is better to identify and define concerns. Reality-based ethical beliefs seek continuity of thought and lasting solutions. Quality of sense experience improves with better tools of observation and measurement with rigorous criteria for validation. Logical relations track consistency of observed and measured (counts, properties) behaviors in a representation. Virtually all forms of representations, ranging from mimicry to math and quantum mechanics, elicit realities useful for use and creation of knowledge. Inference from assertions may be abstracted and expressed with tools of mathematics (measure theory, algebra, geometry, and calculus). Constraints of logic hold for all forms of information, albeit endpoints may differ for theory, machine computation, or processing of biological information. Rules for processing information and relations inherent in a set of affirmed assertions are yet to be developed. These are likely to facilitate development of machines that can think, judge, and make decisions.

\section{Logic Tracks Asserted and Affirmed Reality}

Descriptions of observed and measured behaviors of real objects and concerns can be logically scrutinized and evaluated. Logical objects retain their identity during manipulations for reasoning. They do not disappear into nothing nor do they materialize from nowhere. Sense inputs from external reality are the training set for programming the mind to track reality for reasoning and recall. Viable relations from sense experience of real objects may lead to assertion it is or it exists to be logically confirmed or refuted by weight of arguments and independent evidence, and also by power to predict. Bits and pieces of real world behaviors are objects for 
dreams and fiction but their grotesque images are rarely comprehensible enough to share with others for reasoning. Imaginary assertions may mimic life experiences but a crow can be made to dance like a peacock with out of context bits and pieces.

Affirmed assertions and inference propositions form the basis of discourse and inquiry about sense experience. Empirical knowledge by trial and error relies on inputs from diverse experiences. Observers separated by geography and time verify inputs to seek invariance, stability, and reliability. Constructs of shared experiences are remarkably useful to successfully identify concern, define problem, and address concerns. Those that improve quality of life remain useful for future use and seek out wider participation. Print and internet media have fundamentally changed the way information is disseminated and shared for open discourse. It has unleashed creative expression in ever wider circles of participants. Content on the Web initially may be $60 \%$ reliable. It continues to improve from feedback to correct mistakes and examine nuances to rid of irrelevant and barren ideas.

\section{Bounds of Logic: Affirmed Reality (sat) versus Truth (satya)}

Perceptions that guide decisions, choices, and actions are not exclusively based on reality or truth. Perceptions are not synonymous with truth or reality. As matter of emphasis, perceptions may be inconsistent, contradictory, and changeable. Truth is about $I$ know it to be so, or even I know that it is not so guided by justified beliefs. On the other hand, reality inferred from affirmed assertions seeks certainty, consistency, and non-contradiction as in unequivocal it is so or it exists. It is or it exists is inferred a posteriori from behaviors of identified objects. Inference propositions from Nay reasoning seek convergence with open-ended search for assertions that may surface later. Such inferences are tentatively expressed with may be, it is so to identify and address concerns at each stage of the search to reduce remaining doubt and uncertainty. Valid inference propositions are logically consistent with all affirmed assertions and contradict none. It may or may not be the truth as commonly known, or what has been handed down as testimony from authority, or culled from miracles, faith, self-evident truths, or ad hoc justified as true-beliefs. Superficial and literal interpretation of words for insight in the content of a construct shows ignorance of both. A person named Jesus may not have unique attributes of The Jesus. Also truth values of Saptbhangi Syad propositions have little to do with relativism (Doctrine that knowledge, truth, and morality exist in relation to culture, society, or historical context, and are not absolute) or theory of relativity or uncertainty principle of physics.

Reality of an object or validity of a concern inferred from positive evidence exists independent of the observer. The goal of Nay reasoning (Jain logic) is to represent logical states of sense experience on the basis of available evidence. Reasoning with affirmed assertions about parts and relations leads to propositions about the object or concern as may be, it is so. Such inferences necessarily remain tentative as long as it is likely that additional assertions may surface in the future. Without pretending to predict the future, Nay navigates reasoning through reality based consequences of actions and behaviors. This intellectually honest approach acknowledges liabilities from human fallibility, uncertainties (sanshay) in relationships of input and output, and doubt (syad) in methods and conclusions.

\section{Mathematics and Logic}

Reality perceived by senses may be complex but never contradictory. Both mathematics and logic try to capture key features of reality perceived from sense inputs. Approximation of binary complementation permits 
search for simplicity, beauty, and symmetry required for pruning alternatives. Importance of insights from such mind games is to anticipate essential features of reality as in the theorems of Euclid, Appolonius, and others who paved the way to describe rather complex parts of universe for astronomy, physics, general relativity, and quantum mechanics. It has been surmised that mathematics has been unreasonably effective in describing and anticipating aspects of natural science. Arguably, at times natural sciences have also paved the way for validation of mathematics. Reality is much more complex because descriptions of unified whole from parts require knowledge of all the relations and independent proof for completeness of the set.

Identity of make-believe (dream) and fictional objects are unreal and ephemeral. Their self-referential character cannot be affirmed by independent evidence. Logical and mathematical objects can be affirmed by independent evidence. They retain their identity during manipulation in different contexts, and their content cannot be created from nothing nor disappear into nothing. Both logic and mathematics track observable and measurable relations between real objects to weed out contradictions and resolve inconsistency and paradoxes.

The logic of mathematics is not the mathematics of logic. Both relate to behaviors of real objects. Logic seeks valid relations in articulated sense experience, whereas focus of mathematics is on relations of objects in a representation. Language of mathematic has many dialects that deal with the same reality with different degrees of effectiveness. One of the key assumptions for analysis of relations and their machine implementation is the closure condition that assures balance of inputs and outputs. Nay logic uses independent evidence to affirm each assertion. It is open-ended search for which the closure condition cannot be explicitly stated, except that it is inherent in sense experience and evidence. Functions of mind also follow from the reality perceived by senses and interpreted by the mind trained with the same kinds of inputs to arrive at conclusions consistent with available evidence. Whether or not expressed explicitly, closure is inherent in the constraints of reality used to chart the logic space of a proposition. Logic of inference therefore calls for a kind of mathematics that can deal with systems where closure condition may be approximated.

\section{Binary (Classical Western) Logic}

Virtually all forms of logics, including the binary Western logic, deduce truth value of one of the variables in a proposition in relation to the truth-values of all other variables assumed or be justified true belief, or self-evident, or based on testimony, or past-practices (Suppes 1957). Rules of mathematical deductions follow from the injunctions about a closed system of objects and relations. The power of mathematical deduction lies in the fact that all mathematical injunctions are consistent with each other, and do not contradict real world behaviors. Numbers and symbols in algebra track objects as scalar quantities. With additional assumptions, it can be extended to logic of relations in vector spaces (Mizraji 1992, 1996; Ramachandran 1983; Stern 1988, 1992; Westphal 2005). It is likely that measure theory for logic could be formulated to provide a reasonably complete description of interaction of information with evidence with the assumptions that approximate reality as a closed system. It is still a challenge to minimize remaining doubt in proposition and chart the logic states of an open system that accommodate new inputs.

Complementation of presence versus absence (states) of an object in a given space at a given time is the basis of the closure condition in Boolean algebra and binary (classical Western) logic. It permits logical deductions with two states of a proposition as scalar quantities. The binary states ( 1 or 0 , true or false, present or absent) ignore the intermediate states (excluded middle) of partial truth value (probabilities, fuzzy states). It 
follows that if the object exists but not-present (not-true, not 1), it is necessarily absent (false, 0 ); also if it is not-absent (not-false, not 0 ), then it is present (true). Such self-referential relationship between $\mathrm{T}$ and $\mathrm{F}$ leads to contradictions and paradoxes (Smullyan 1987; Smullyan 1980). With evidence for one state (say 1), the other state $(0=$ Not- 1$)$ is deduced with the connective Not. Such states of variables may also be related through connectives OR \& AND. All relations of one (unary) or two (binary) inputs to one output are thus expressed as operations with three connectives (NOT, AND, OR), or with NAND (not-AND) or NOR (not-OR). Technological developments to rapidly manipulate large number of switches in ON (1) or OFF (0) states allow machine implementation of circuits for computation with suitably configured NAND gates for Boolean deduction and other logic operations. Multiple states of inferences with different (partial) truth values from Nay reasoning have not been machine implemented yet.

\section{Context-Dependent Meanings of Negation (NOT)}

In common usage, the connective NOT is prefixed as in not-present. It is interpreted to indicate absence of an object that exists and was present before in the given space and time. In a general and idealized sense, NOT relates two states that are polar opposite in every possible way. Not-present negates the presence of an object (noun) that exists, but absence of the object provides little information about the object. In binary complementation, it is difficult to decide what is meant by Not-T. If $\mathrm{T}$ is nothing but truth, multiple not-true states will have range of partial truth values. Are they all false? Negations characterize opposite, reverse, contrary, not-the-same, or different states. Context-dependent relations or properties of adjective, verb or adverb are often negated with different emphases with prefixes like un-, non-, a-, mis-, dis-, im-, in-. Is an ignorant person not-informed, uninformed, or misinformed? Terms like complement, transpose, and conjugate have specific meaning for operations in mathematics. True (1) may be a single state that exists, whereas not-true is for everything else. Such considerations preclude representation of states negated with logical NOT. These are certainly not transforms. The proposition if $p$ then $q$ (reverse implication) may also be interpreted as: if not- $q$ then not- $p$ (counterpositive), or if not- $p$ then not- $q$ (inverse), or if $q$ then $p$ (converse). Proposition if $p$ then $q$ is interpreted as $p$ implies (reverse or forward) $q$ (in logic), or $\mathrm{p}=\mathrm{q}$ in algebra where equality retains the intended meaning with $q=p$ for reverse implication, but not permitted with forward implication. Interpretation of negations in not $-p=$ not $-q$, or not $($ not $-p)=q$, or $q=$ not (not- $p$ ) for implications is not trivial. Closure condition by complementation permits binary deduction of present as not-(not-present), as with a two-way switch turned off and then on again.

A major concern of the Jain Nay is non-existence which is not the same as absence of an object that exists. Nonexistent object can neither be present nor absent. Also lack of evidence for presence of an object is not the evidence for its absence, or for its nonexistence. Independent evidence is required to assert absence, and also for non-existence. Ignorance often forces a conclusion based on closure with ad hoc, hidden, or incomplete set of assumptions. Such self-reference invariably leads to paradox of $\mathrm{p}=$ not-p. Syad Nay avoids such contradictions by negation of existence as null for nonexistent interpreted as indeterminate state on the basis of available evidence (see below).

\section{Other Limitations of Binary Logic}

Binary complementation, without independent evidence for each state, results in loss of information about the state that is neither true nor false (indeterminate), or is both true and false (see below). Both states are in 
fact assigned on the basis for one: If true (present) is based on evidence, false (absent) would follow from the lack of evidence for true. It amounts to self-reference. Complementation of 0 and 1 states assumes complete set of inputs as a basis for the closure condition. It is also necessary for deduction of truth tables for other logical connectives (operators). In binary logic, the inputs and connectives take the form of an equation to describe the behavior of assertions $p, q$, and $r$ in a proposition:

$$
\begin{aligned}
& \text { If } p \text { AND } q \text { then } r \quad \text { (or } p \text { AND } q \text { imply } r \text { ) that is } p=1 \text { AND } q=1, r=1 \\
& \text { Or } p=0 \text { AND } q=0, r=0 \\
& \text { Or } p=0 \text { AND } q=1, r=0 \\
& \text { Or } p=1 \text { AND } q=0, r=0
\end{aligned}
$$

Unlike the equality in arithmetic or algebra, the logical (forward) implied equivalence (equality) is not reversible or invertible. Truth table for the AND connective in $\mathrm{p}$ AND $\mathrm{q}=\mathrm{r}$ has relations (above) of $\mathrm{r}$ not only to $p$ and $q$, but also to not-p and q, not-p and not-q, and not-p and not-q. Now try making the truth table for the negated proposition $p$ AND $q$ then NOT-r, or for NOT (p AND q) then r. A major difficulty in casting the mathematics of binary logic lies in the fact that the truth-tables of binary operators AND \& OR are not reversible (invertible). Under certain conditions, it results in loss of information about other possible states. States $\mathrm{T}$ or $\mathrm{F}(1$ or 0 ) for $\mathrm{p}$ can be obtained from the truth value of $\mathrm{q}$ and $\mathrm{r}$ : $\mathrm{r}$ is 1 only if both $\mathrm{p}$ and $\mathrm{q}$ are 1 , and $\mathrm{r}=0$ for the other three cases where either $\mathrm{p}$ or $\mathrm{q}$ or both are 0 . For $\mathrm{r}=0$ and $\mathrm{p}=0$ or 1 , $\mathrm{q}$ can be 0 or 1 (doubtful or D state).

\section{Saptbhangi Syad Nay}

To circumvent some of the limitations of complementation, a Nay assertion is affirmed by independent evidence, and its negation can only be affirmed with separate and independent evidence. A proposition with multiple assertions can have multiple states: $\mathrm{N}$ assertions give $2^{\mathrm{n}}$ states of proposition with different truth values. Two additional states of a proposition emerge: In addition to orthogonal $\mathrm{T}$ and $\mathrm{F}$ (as in the square diagram), there is the doubtful (D) and indeterminate (X) states of a proposition with two assertions, it exists and it does not exist. Both assertions are affirmed in D, and neither affirmed in X. With additional assertions, there would be only one $\mathrm{T}$ and one $\mathrm{X}$ state. More than 1 orthogonal $\mathrm{F}$ states and many more $\mathrm{D}$ states with partial truth values are possible. These arguments were probably first formulated by Bhadrabahu I (ca. 350 BC), and used later by others to argue against nothingness, the Buddhist idea of shoonyata (as X or null), and all-inclusive Brahm of eti eti, neti neti (it is this and it is also not this) as doubtful (D).
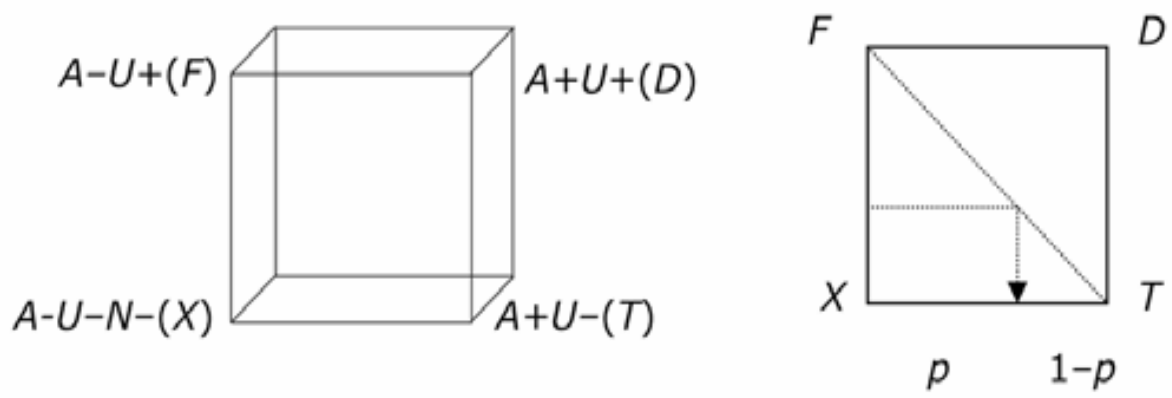
Table 1

Propositions with Three Assertions Affirmed (+) or Not Affirmed (-): Does Not Exist (N), Exists (A), Undescribable or Indescribable (U)

\begin{tabular}{lllll}
\hline & $\begin{array}{l}N \\
\text { (does not exist) }\end{array}$ & $\begin{array}{l}\text { A } \\
\text { (exists) }\end{array}$ & $\begin{array}{l}\text { U } \\
\text { (undescribable) }\end{array}$ & Bit map \\
\hline 1 & - & - & - & 000 \\
2 & - & + & - & 010 \\
3 & - & - & + & 001 \\
4 & - & + & + & 011 \\
5 & + & - & - & 100 \\
6 & + & + & - & 110 \\
7 & + & - & + & 101 \\
8 & + & + & + & 111 \\
\hline
\end{tabular}

Sense inputs provide information about the identity of objects and additional information follows from the evidence about its content and context. Mind also seeks criteria- and evidence-based consistency of sense inputs to rule out contradictions. Objects amenable to sense experience can be represented (satprarupana) by gathering additional generalizations (anugam). What, when, where, how type of questions provide additional insights about its class, shape, size, distribution, and tendencies? Definitions in modern science are also descriptions of observable and measurable quantities. Note that descriptions based on negations alone are meaningless, such as formless, forever, everywhere, beyond sense experience etc.

Inference proposition with multiple assertions minimizes the remaining liabilities (syad). There are four possible states for a proposition with two assertions $\left(2^{2}\right)$, and $2^{3}$ possible states with 3 assertions. The logic space for two orthogonal assertions is a square (or circle) in a 2-dimensional plane, and a 3-dimensional cube (or a sphere) for a proposition with three orthogonal assertions. Each corner (node) of square or cube corresponds to a distinct logic state of the proposition. One of these is null X (indeterminate state) state with no affirmed assertions which implies non-existence for all intents and purposes. The other seven have unique truth values determined by 1, 2, or 3 assertions affirmed by independent evidence. The three orthogonal states in the classical example for Saptbhangi are: It exists and it is observable; it exists but it is undescribable; and it does not exist because consequences of its presence or absence are not different.

We may not be able to perceive or draw n-dimensional space but logically and mathematically, it is real with $2^{\mathrm{n}}$ nodes for as many states. Two or three dimensional space can also be represented in polar coordinates. For the present purpose, the Cartesian representation is intuitive because human senses perceive, interpret, and reconstruct the world in 1, 2, and 3 dimensions with different assumptions. The logic space of a proposition can have as many dimensions as needed for representation and abstraction. Certainty of a proposition increases with the number of affirmed orthogonal assertions, and assertions not affirmed by evidence may be interpreted for consistency with other affirmed assertions. Convergence to the reality with nothing but absolute truth value (keval) occurs with virtually infinite number of orthogonal assertions, each affirmed by independent evidence. Reasoning with affirmed assertions requires an identified object or concern with defined content and context. Each independent affirmed assertion as an orthogonal vector contributes a dimension to the logic space. Three mutually perpendicular orthogonal vectors with normalized magnitudes form a symmetrical cube as the logic space of Saptbhangi propositions for three assertions connected with AND. NOT relates orthohgonal assertions that negate each other. The logic space of a proposition with three assertions represented as cube has eight 
distinct states that correspond to the corners of the cube. As summarized in the Table above, each of the seven Saptbhangi propositions with 1, 2, or 3 affirmed assertions has distinctly different deterministic truth values. The eighth state of the proposition with no affirmed assertion is for the null node interpreted as nothingness (shoonyata) in which none of the three assertions is affirmed. Note that absence is interpreted as NOT-present in binary logic however relationship of existence to non-existence is much more subtle and nuanced.

\section{Inference with Multiple Assertions}

Advent of quantum mechanics has brought into focus the need to reason with multiple assertions about the logic states of a proposition. Formalism of vector-matrix algebra has proven to be remarkably useful to account for these quantum states. The crux of the formalism for the purpose of logical reasoning is that the possible logic states are the consequence of a set of orthogonal vectors which may be expressed as matrix. In this formalism, an assertion vector for it is or it exists (true) is orthogonal to its negation vector it is not or does not exist (false). Independent evidence is required for assertion of it is not as an orthogonal vector.

Vector matrix formalism for the logic space of two orthogonal basis vectors can express all Boolean functions in terms of vector matrix algebra. In this representation, both $\mathrm{T}$ and $\mathrm{F}$ states are affirmed by independent evidence, and much of the logic space is for $\mathrm{D}$ states which are discarded in classical binary logic as excluded middle. In search of additional inputs, its conclusions are always incomplete and tentative. Could this be the structure of mental images? A tentative image may be formed with a very limited set of vectors. Each additional affirmed assertion provides additional vector to enhance the mental age of the representation as proposition. Such exploration and discovery is a part of individual as well collective learning, where propositions morph into abstract objects in Hilbert space bounded by orthogonal vectors culled from independent inputs (sense experiences). It will be interesting to examine if neural circuits of brain use such matrices for processing defined sets of inputs and outputs for specific purposes as already demonstrated for physical devices (outlined next).

\section{Logic Gates}

The 2D space bounded by two orthogonal vectors (01 and 10) provides a basis to represent not only the binary states but also the D (doubtful, unresolved), $\mathrm{X}$ (indeterminate) for null state as shown in the square above. The T-F diagonal provides ways to represent and interpret range of probabilities as a slice of partial truth values between $\mathrm{T}$ and $\mathrm{F}$. Such formalisms with suitable assumptions and approximations can be adopted with different degree of success for the various logics (Fuzzy, Bayesian) and statistics implemented in binary computers.

The 3x8n matrix in the last column in the Table above is the Truth Table of 3 bits inputs. A similar table exists for 3 bits of output (not shown). Both the matrices can be trimmed and configured for specific purposes. Reversible and controlled 3 bit gates for binary and quantum computing have been constructed: Toffoli gate is for controlled-controlled-not (CCNOT); Fredkin gate is for controlled swap. The logic of vector space is open-ended because it can accept new vectors and trim others, and then reconfigured. Circuits, networks, and physical devices with bits for 3 or more inputs have been developed to provide single logical output. Such logic gates are used to implement electronic switches (diodes or transistors) and relays for the various kinds of logics, and more complex devices such as multiplexers, registers, arithmetic logic units, computer memory, and microprocessors. These logic networks and gates can be cascaded to Boolean functions with more explicit 
algorithms and mathematics. Considerable effort is invested for perfecting quantum gates into practical devices. Their feasibility is now well established. Difficulty seems to be in the implementation of quantum networks into functional circuits with multiple simultaneous inputs in quantum bits (qbits). Their main advantage is that all of the states of the system coexist. Such devices with more qbits are expected to be faster than the binary devices; $2^{10}(=1024)$ fold faster for a device with 10 qbits.

\section{Perpetual Doubt of Human Condition}

As organisms overcome challenges of the past as they cope with the demands of the present and learn to steer through uncertain future by trial and error. States of doubt encountered along the way are necessary part of perpetual human condition because the future is neither predetermined nor predictable. It is futile to search for omniscience or the ultimate reality or truth. It is practically impossible to write an analytical equation of universe to predict past, present, and future, or to predict the future on the basis of the chain of action-consequence relations. Unknowable cannot be known, but it is useful to know the unknowable that introduce doubt.

Chances of success increase as sources of doubt are identified and addressed with independent measures of information and evidence. Ma root in praman (Sanskrit) or paman (Prakrit) refers to independent measures of information as evidence to evaluate validity of sense experience and perceptions. Rules of logic and algorithms for processing information and evidence are likely to be universal if they track real world behaviors. Mathematical formulation of such rules under binary limiting conditions has already made it possible to implement deduction for computation. Inferences from the natural information processing systems are for real time approximations from multiple inputs. Since it is carried out by neural circuits, it should be possible to do with electronic circuits. Detailed layout of neural networks for specific functions and parallel processing remain to be worked out. Understanding mind, intelligence, and other functions of brain would require relating biophysics of single neurons to computation algorithms for memory and recall in the context of a theory of learning. Such understanding could ultimately pave the way for intelligent machines that make decisions and judge consequences. It may not be trivial. A far more difficult task for human intelligence would be to judge potential risks of such artificial intelligence and the decide how to successfully coexist with it.

\section{Works Cited}

Hiraiynna, M. Outlines of Indian Philosophy. London: George Allen \& Unwin Ltd., 1932.

Jain, M. K. “Logic of Evidence Based Inference Propositions.” Current Science 100 (2011): 1663-72.

Mizraji, E. "Vector Logics: The Matrix-Vector Representation of Logical Calculus.” Fuzzy Sets Syst. 50 (1992): 179-85.

---. “The Operators of Vector Logic.” Mathematical Logic Q. 42 (1996): 27-40.

Ramachandran, G. N. "Vector-Matrix Representation of Boolian Algebras and Application to Extended Predicate Logic (Part I \& II).” Current Science 52 (1983): 292-302; 335-41.

Rogers, J. M. and M. K. Jain. “Inference and Successful Behavior.” Quarterly Rev. Biol. 68 (1993): 387-97.

Roy, A. K. A History of the Jainas. New Delhi: Gitanjali Publishing House, 1984.

Saha, S. B. "Shatkhandagam of Pushpadant and Bhutbali (ca. 35 AD) with Introduction by H. L. Jain.” Faltan: Jain Jinvani Jeernodhhar Sanstha, 1965. 840.

Sanghvi, S. The Four Tirthankars. Varanasi: Prshvanath Vidyashram, 1989.

Smullyan, R. Forever Undecided: A Puzzle Guide to Godel. New York: Knopf, 1987.

---. This Book Needs No Title: A Budget of Living Paradoxes. Engelwood Cliffs: Prentice Hall, 1980.

Stern, A. Matrix Logic. Amsterdam: North Holland, 1988. 
---. Matrix: Logic and Mind. Amsterdam: Elsevier, 1992.

Suppes, P. Introduction to Logic. Princeton: D. Van Nostrand Co., 1957.

Tatia, N. "That Which Is (Tatvarth Sutr of Umaswami ca 200 AD).” San Francisco: Harper Collins Publ., 1994.

Westphal, J. a. H., J. “Logic as a Vector System.” J. Logic Computat. 15 (2005): 751-65. 